\title{
A fornix infarction causing post-operative anterograde amnesia
}

\author{
Shyh Poh Teo ${ }^{\mathrm{a}, *}$ \\ ${ }^{a}$ Geriatrics and Palliative Unit, Department of Internal Medicine, Raja Isteri Pengiran Anak Saleha (RIPAS) Hospital, Ja- \\ Ian Putera Al-Muhtadee Billah, Bandar Seri Begawan BA1710, Brunei Darussalam.
}

\begin{abstract}
A 65-year-old woman underwent elective hip replacement surgery and postoperatively developed significant anterograde amnesia, without other significant neurological deficits. Magnetic resonance imaging (MRI) revealed an area of infarction in the anterior fornix and genu of the corpus callosum. Neuropsychological testing revealed extremely poor learning performance and immediate memory, as well as delayed memory, consistent with anterograde amnesia. This case illustrates the relationship between the anterior fornix and memory function and the role of hypoperfusion brain injury in ischemic strokes.
\end{abstract}

Keywords: Anterograde amnesia, brain fornix, ischaemic stroke, postoperative period

\section{Introduction}

A stroke following total joint arthroplasty is rare, occurring in around $0.2 \%$ of patients [1]. The first 2 weeks after arthroplasty are high risk in terms of developing an ischemic stroke, with almost a five times greater risk compared to that in an age-adjusted population. The risk remains high for about 6 weeks, with the greatest risk occurring in the immediate postoperative period [2]. Cerebral hypoperfusion, marrow embolization, and anesthetic effects on the cardiovascular system have been cited as possible mechanisms underlying this ischemic risk [1,2]. Ischemic strokes affecting the anterior fornix are also an infrequent occurrence, with just a few case reports published [3-8]. All these case reports demonstrated a link between acute amnesia and fornix infarcts. The role of the anterior fornix in memory function is related to its anatomical placement within the Papez circuit, which comprises the fornix, hippocampus, mammillary bodies, anterior thalamus, and cingulate gyrus $[9,10]$. The anterior fornix acts as the output tract from the hippocampus, primarily to the mammillary bodies. The primary function

\footnotetext{
* Corresponding author: Shyh Poh Teo

Mailing address: Geriatrics and Palliative Unit, Department of Internal Medicine, Raja Isteri Pengiran Anak Saleha (RIPAS) Hospital, Jalan Putera Al-Muhtadee Billah, Bandar Seri Begawan BA1710, Brunei Darussalam.

Email: shyhpoh.teo@moh.gov.bn

Received: 13 May 2021 / Accepted: 29 May 2021
}

of the Papez circuit, which was first described in 1937, involves encoding and recall of new, episodic information. Memory function is more reliant on the structural integrity of the circuit as a whole rather than the integrity of each of the individual components $[9,10]$.

In this paper, a case of a patient who developed a postoperative fornix infarct and anterograde amnesia is described, followed by a discussion of the relationship between the anterior fornix and memory function and the role of hypoperfusion brain injury in ischemic strokes.

\section{Case Report}

Ms. O was an independent 65-year-old admitted for an elective right total hip replacement for osteoarthritis. She had a background of hypertension and hyperlipidemia and was receiving alpha-blocker, beta-blocker, and statin therapies. There were no other risk factors for strokes. During the intraoperative and immediate postoperative period, she became hypotensive with lowest intraoperative blood pressure being 80/40 despite cessation of antihypertensive drugs, and required fluid resuscitation. During the first 24 hours post-surgery, she appeared confused and disoriented, without focal neurological deficits. She repeatedly asked where she was and why she was in hospital, even after she was reorientated by nursing staff. A computed tomography (CT) brain (Figure 1A) 48 hours after surgery showed hypodensity in the medial aspect of the frontal lobe, appearing to involve the anterior corpus callosum. Brain magnetic resonance imaging (MRI) (Figure 1B) revealed an area of increased signal in fluid-attenuated 


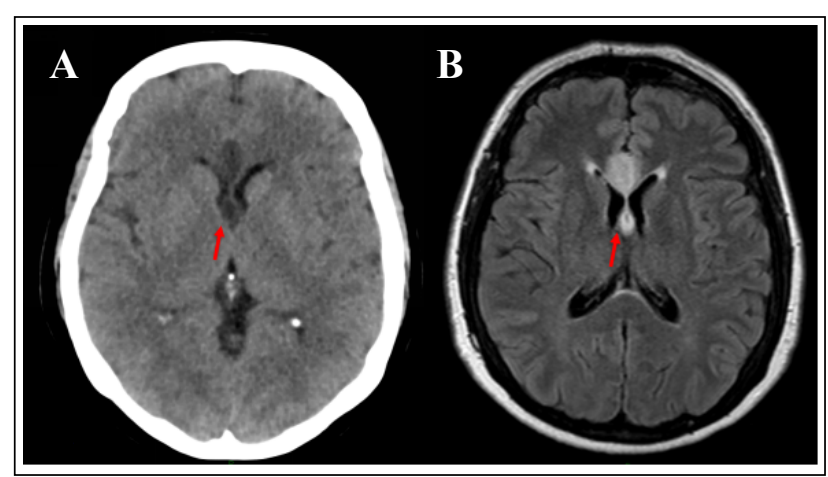

Figure 1. Brain imaging showing the fornix infarct (A: CT; B: MRI FLAIR).

inversion recovery (FLAIR) and diffusion-weighted imaging (DWI) sequences in the beginning of the corpus callosum, suggesting acute diffusion impairment in the genu of the corpus callosum, extending into the fornix and septum pellucidum. A diagnosis of an acute fornix infarction was made.

A neuropsychological assessment, including the Repeatable Battery of the Assessment of Neuropsychological Status, revealed extremely low performance in two areas: learning and immediate memory and delayed memory. These results were incongruent with the remainder of the patient's test scores of average or higher for premorbid ability, attention, processing speed, visuospatial and constructional ability, fluency, and insight. The patient was later transferred to an inpatient brain injury rehabilitation service and required 3 months of multidisciplinary brain rehabilitation before discharge to the community.

\section{Discussion}

The case of a patient who developed postoperative anterograde amnesia due to a fornix infarct was described. This case reveals the relationship between the anterior fornix and memory function, as well as the role of hypoperfusion brain injury in ischemic strokes. Acute fornix infarcts are rare, and previous case reports describe acute amnesia as the main presenting symptom [3-8]. Two of these cases were due to an isolated fornix infarction $[3,4]$, while the other cases [5-8], including the one described herein, were associated with ischemia of nearby structures, including the corpus callosum and cingulate gyrus. Interestingly, the case of the isolated left anterior fornix injury [4] was not clinically different to the case with the isolated bilateral fornix infarction [6], suggesting that isolated fornix lesions can cause amnesia and a lack of laterality to fornix function. This may be due to the dual role of the fornix as a tract from the thalamus to the basal forebrain and as a commissure between the left and right hemispheres [9]. Multiple pathologies can damage the anterior fornix, either on their own or as part of a diffuse process. These include congenital absences, neoplastic diseases, infections, autoimmune diseases, metabolic diseases, infarctions, and traumas. Isolated fornix infarcts almost exclusively occur in the anterior columns. This is likely due to the proximity of the remaining fornix to the choroidal arterial supply aiding collateral perfusion and protecting against infarctions [9]. Blood to the anterior fornix is supplied by branches of the anterior communicating artery (ACoA) [9], which accounts for the high rate of postoperative amnesia in ACoA aneurysm surgery $[11,12]$. Other variations to the vascular supply that can cause anterior fornix infarctions include occlusion of the subcallosal artery [8] or the anterior cerebral artery via short medial central arteries [3]. Approximately $20-50 \%$ of poststroke patients have subjective complaints of memory difficulties, which may be accounted for by nonanatomical etiologies, such as depression, fatigue, medication side-effects, or sleep disorders. However, lesions within memory-associated structures or amnesic lesions and lesions impairing memory processing (process lesions) should be considered [10]. Pathological processes affecting hippocampal and diencephalic connections are consistently more likely to impair memory function than fornix lesions [10,13]. Interestingly, in a case study on 142 epileptic patients who underwent bilateral anterior fornix transection, no patients experienced long-term memory impairment [14].

In the present case, the fornix infarct occurred during the perioperative period. Hypoperfusion of the fornix during the period of hypotension contributed to the infarct in this case. The combination of systemic hypotension and preexisting extracranial arterial occlusive disease caused a reduced blood supply, which led to a hemodynamic stroke [15]. In this patient, carotid Doppler ultrasound revealed $50-69 \%$ stenosis in the right internal carotids. Although this level of stenosis is considered only mild-to-moderate, the concurrent hypotension likely contributed to the hypoperfusion and the hemodynamic stroke. Hypotension is a known cause of ischemic strokes, with a high risk among those with symptomatic orthostatic hypotension and cardiac failure [15]. Intraoperative blood pressure has been shown to be directly related to the neurological outcome following cardiac surgery, with almost $50 \%$ of patients undergoing cardiac surgery having MRI evidence of bilateral ischemic lesions in "border zone" areas between the main brain vascular supplies, which are vulnerable areas to ischemic injury. [16] The risk of watershed infarcts was higher in patients with an intraoperative fall in systolic blood pressure of $10 \mathrm{mmHg}$ or more [16]. Another study found radiological evidence of strokes in $7.2 \%$ of patients with intraoperative mean arterial pressure of 50-60 $\mathrm{mmHg}$ as compared to $2.4 \%$ when mean arterial pressure was maintained between 80 and $100 \mathrm{mmHg}$ [17]. Thus, maintaining blood pressure and brain perfusion during the intraoperative and postoperative periods is important to reduce the risk of ischemic strokes.

In the present case, the patient required an intensive multidisciplinary assessment and treatment at an inpatient specialized acute brain injury rehabilitation service. The patient was discharged after 3 months of inpatient rehabilitation. Early initiation of postacute rehabilitation treatment in brain injury is beneficial and increases the likelihood of a functional improvement [18]. The mechanism 
underlying such recovery is likely multimodal, ranging from controllable factors (motivation, family support systems, and rehabilitation service quality) to uncontrollable factors (age, sex, injury severity and site, and premorbid cognitive reserve) [19].

The long-term cognitive outcome after acute fornix infarcts is uncertain. One study described a patient who developed amnesia and confabulation following astrocytoma removal, which disrupted the right anterior fornix. [13] This patient made a good recovery over a 17-month follow-up period, suggesting that recovery is possible in the long term. Compensatory measures by the left anterior fornix likely account for recovery in such patients [13]. However, a remaining functional fornix is not a prerequisite for memory recovery, as observed in patients who improved cognitively after bilateral fornix transection [20].

\section{Conclusion}

A case of a fornix infarct resulting in anterograde amnesia was described. The fornix infarct was likely due to a hemodynamic stroke caused by perioperative hypotension and hypoperfusion. The patient required brain rehabilitation and recovered sufficiently to be discharged after 3 months. However, the long-term outcome in terms of cognitive recovery remains unclear.

\section{Declarations}

Authors' contributions: The author contributed solely to the article.

Conflict of interest: The author declared that there are no conflicts of interest.

Consent for Publication: Written informed consent for publication of de-identified case details and radiological images were obtained from the patient (witnessed by the son).

\section{References}

1. Mortazavi S M J, Kakli H, Bican O, et al. Perioperative stroke after total joint arthroplasty: prevalence, predictors, and outcome. JBJS, 2010, 92(11): 2095-2101.

2. Lalmohamed A, Vestergaard P, Cooper C, et al. Timing of stroke in patients undergoing total hip replacement and matched controls: a nationwide cohort study. Stroke, 2012, 43(12): 3225-3229.

3. Moudgil S S, Azzouz M, Al-Azzaz A, et al. Amnesia due to fornix infarction. Stroke, 2000, 31(6): 1418-1419.

4. Korematsu K, Hori T, Morioka M, et al. Memory impairment due to a small unilateral infarction of the fornix. Clinical neurology and neurosurgery, 2010, 112(2): 164-
166.

5. Renou P, Ducreux D, Batouche F, et al. Pure and acute Korsakoff syndrome due to a bilateral anterior fornix infarction: a diffusion tensor tractography study. Archives of neurology, 2008, 65(9): 1252-1253.

6. Rizek P, Pasternak S, Leung A, et al. Acute-onset anterograde amnesia caused by isolated bilateral fornix infarction. Canadian journal of neurological sciences, 2013, 40(5): 738-739.

7. Saito Y, Matsumura K, Shimizu T. Anterograde amnesia associated with infarction of the anterior fornix and genu of the corpus callosum. Journal of Stroke and Cerebrovascular Diseases, 2006, 15(4): 176-177.

8. Moussouttas M, Giacino J, Papamitsakis N I H. Amnestic syndrome of the subcallosal artery: a novel infarct syndrome. Cerebrovascular Diseases, 2005, 19(6): 410.

9. Lim C, Alexander M P. Stroke and episodic memory disorders. Neuropsychologia, 2009, 47(14): 3045-3058.

10. Thomas A G, Koumellis P, Dineen R A. The fornix in health and disease: an imaging review. Radiographics, 2011, 31(4): 1107-1121.

11. Gade A. Amnesia after operations on aneurysms of the anterior communicating artery. Surgical neurology, 1982, 18(1): 46-49.

12. Hattingen E, Rathert J, Raabe A, et al. Diffusion tensor tracking of fornix infarction. Journal of Neurology, Neurosurgery \& Psychiatry, 2007, 78(6): 655-656.

13. Ruggeri M, Sabatini U. Recovery from amnesic confabulatory syndrome after right fornix lesion. Neurorehabilitation and neural repair, 2008, 22(4): 404-409.

14. Garcia-Bengochea F, Friedman W A. Persistent memory loss following section of the anterior fornix in humans. A historical review. Surgical neurology, 1987, 27(4): 361364.

15. Klijn C J M, Kappelle L J. Haemodynamic stroke: clinical features, prognosis, and management. The Lancet Neurology, 2010, 9(10): 1008-1017.

16. Gottesman R F, Sherman P M, Grega M A, et al. Watershed strokes after cardiac surgery: diagnosis, etiology, and outcome. Stroke, 2006, 37(9): 2306-2311.

17. Gold J P, Charlson M E, Williams-Russo P, et al. Improvement of outcomes after coronary artery bypass: a randomized trial comparing intraoperative high versus low mean arterial pressure. The Journal of thoracic and cardiovascular surgery, 1995, 110(5): 1302-1314.

18. Hayden M E, Plenger P, Bison K, et al. Treatment effect versus pretreatment recovery in persons with traumatic brain injury: a study regarding the effectiveness of postacute rehabilitation. PM\&R, 2013, 5(4): 319-327.

19. Wilson B A. Brain injury: recovery and rehabilitation. Wiley Interdisciplinary Reviews: Cognitive Science, 2010, 1(1): 108-118.

20. Mazarakis N K, Summers F, Murray A D, et al. Partial recovery from amnesia following bilateral surgical fornix transection is correlated with cortical plasticity. British journal of neurosurgery, 2011, 25(5): 658-661.

Cite this article as: Shyh P T. A fornix infarction causing post-operative anterograde amnesia[J]. Aging Pathobiology and Therapeutics, 2021, 3(2): 32-34. 